\title{
Achievable Information Rates of Square MQAM Modulation Formats after Carrier Phase Estimation
}

\author{
Milen Paskov*, Domanic Lavery, Alex Alvarado and Polina Bayvel \\ Optical Networks Group, Dept. of Electronic \& Electrical Engineering, University College London, UK \\ *m.paskov@ee.ucl.ac.uk
}

\begin{abstract}
The performance of a pragmatic carrier phase estimation algorithm is evaluated over a range of SNRs. The optimal SNR regions for MQAM are compared to an AWGN channel observing gains of up to $40 \%$ in throughput.

OCIS codes: 060.1660 - Coherent communications, 060.2330 - Fiber optics communications.
\end{abstract}

\section{Introduction}

Digital coherent systems are now widely being adopted to increase capacity of optical fibre networks. Coherent receivers together with analogue-to-digital converters allow for the entire optical field to be translated into the digital domain where transceiver and fibre impairments can be mitigated with Digital Signal Processing (DSP). This enables the use of higher order modulation formats like 16, 64 and 256QAM (MQAM) to further increase system throughput. One of the challenges with higher order modulation formats is the decreased phase margin, which enforces stricter requirements on the laser phase noise and the Carrier Phase Estimation (CPE) algorithm. Often these algorithm are investigated and compared based on their phase noise tolerance [1,2]. However, the performance depends also on the signal-to-noise ratio (SNR) [3,4] and the CPE optimization. Nevertheless, in practical transceivers flexible CPE might not be pragmatic as this would require additional functionality or changes to the DSP chip, which would lead to sub-optimal performance.

In an Additive White Gaussian Noise (AWGN) channel there are well defined SNR regions where the each modulation format is optimal in terms of maximising the information throughput [5]. However, once a practical CPE is used to compensate the laser phase noise these SNR regions are shifted. In this work we demonstrate the loss in Achievable Information Rate (AIR) when CPE is not considered and illustrate how the regions could change for QPSK, 16, 64 and 256QAM, when a Decision-Directed Phase-Locked Loop (DD-PLL) is used to compensate the laser phase noise.

\section{Phase noise}

Laser phase noise originates from imperfections of the resonant cavity, which leads to frequency variation characterised as linewidth. The random process of the laser phase noise can described by a "random walk" (Wiener process [6]) where the difference between two time consecutive points is Gaussian distributed with a variance $\left(\sigma_{\Delta v}^{2}=2 \pi \Delta v T\right)$, proportional to the laser linewidth $(\Delta v)$ and the time between observations $(T)$. Phase noise is also generated through non-linear fibre transmission and can be modelled in a similar way, but this is outside the scope of this work.

There are several different algorithms that can be used to track phase noise [1], and they vary in computational complexity, suitability for hardware implementation, robustness to modulation format change and phase noise tolerance. The common factor between CPE algorithms is that they try to estimate the phase over a symbol sequence (window). The underlying assumption is often that over the window the phase change is linear and by averaging over the multiple symbols the AWGN can be mitigated and a better phase estimate can be achieved. As the phase noise increases the window needs to be decreased as the phase noise is linear over a shorter period, however, this reduces tolerance to the AWGN and a trade-off must be made. After the CPE algorithm a phase inaccuracy still remains as any estimator is limited by the Cramér-Rao lower bound [7]. If this inaccuracy is larger than the phase margin of the modulation format symbol errors can occur. The phase margin for decreases with the modulation order; QPSK, 16, 64 and 256QAM have a margin of $45^{\circ}, 16.9^{\circ}, 7.7^{\circ}$ and $3.7^{\circ}$ respectively.

\section{Simulations configuration}

To illustrate the effect of residual phase noise after CPE, simulations using QPSK, 16, 64 and 256QAM were carried out. The signal was generated at $12.5 \mathrm{GBd}$ and filtered with a root-raised cosine filter with a roll-off of $1 \%$. The signal was then noise loaded with AWGN and phase noise was emulated with a Wiener process and variance of $\sigma_{\Delta v}^{2}$. At the receiver, matched filtering was applied to the signal before the CPE algorithm was applied. In this work a DD-PLL with a loop delay of one symbol was used to estimate the phase noise. The algorithm is described by:

$$
\phi_{k}=\phi_{k-1}+\angle\left(\sum_{n=k-w / 2}^{k+w / 2} D[x(n)] x(n)^{*}\right)
$$


where $\phi_{k}$ denotes the phase at instance $k, w+1$ is the window length, $x(n)$ are symbols de-rotated by $\phi_{k-1}$ and $D[]$ is decision on the symbol. Although this algorithm is not practical for hardware implementation, due to zero delay feedback, it has high tolerance to phase noise [6] and is used to demonstrate the trade-off between window length and SNR tolerance. Additionally it has a simple extension to a more pragmatic feed-forward blind-phase search [3].

To evaluate the trade-offs and find the optimal SNR regions for different MQAM formats, the performance was measured in terms of Generalised Mutual Information (GMI) between the transmitted bits and the received symbols. The GMI is an AIR when considering bit interleaved coded modulation [8]. After CPE the SNR of the signal was estimated and used to calculate log-likelihood ratios (LLRs) utilised for GMI calculations assuming circularly symmetric Gaussian noise statistics [8, eq. 30].

$$
G M I \approx m-\frac{1}{n_{s}} \sum_{k=1}^{m} \sum_{l=1}^{n_{s}} \log _{2}\left(1+e^{(-1)^{c_{k} l}} \lambda_{k, l}\right)
$$

$$
\lambda_{k}=\log \frac{\sum_{x \in \chi_{k}^{1}} \exp \left(-\frac{|y-x|^{2}}{\sigma_{Z}^{2}}\right)}{\sum_{x \in \chi_{k}^{0}} \exp \left(-\frac{|y-x|^{2}}{\sigma_{Z}^{2}}\right)}
$$

where $m$ is the number of bits, $n_{s}$ are the noise samples, $c_{k, l}$ are the transmitted bits and $\lambda_{k, l}$ are the LLR-values computed using the estimate noise $\sigma_{Z}^{2}$. Although LLRs can take into account the residual phase noise information about the linewidth and the applied CPE algorithm would have to be know in advance [9] or the multidimensional estimation has to be carried out [10]. To avoid any noise outliers and cycle slips which can corrupt an entire data set (if they are not corrected), each performance point was averaged over 30 completely independent realizations with $2^{15}$ symbols giving an estimate on AIR.
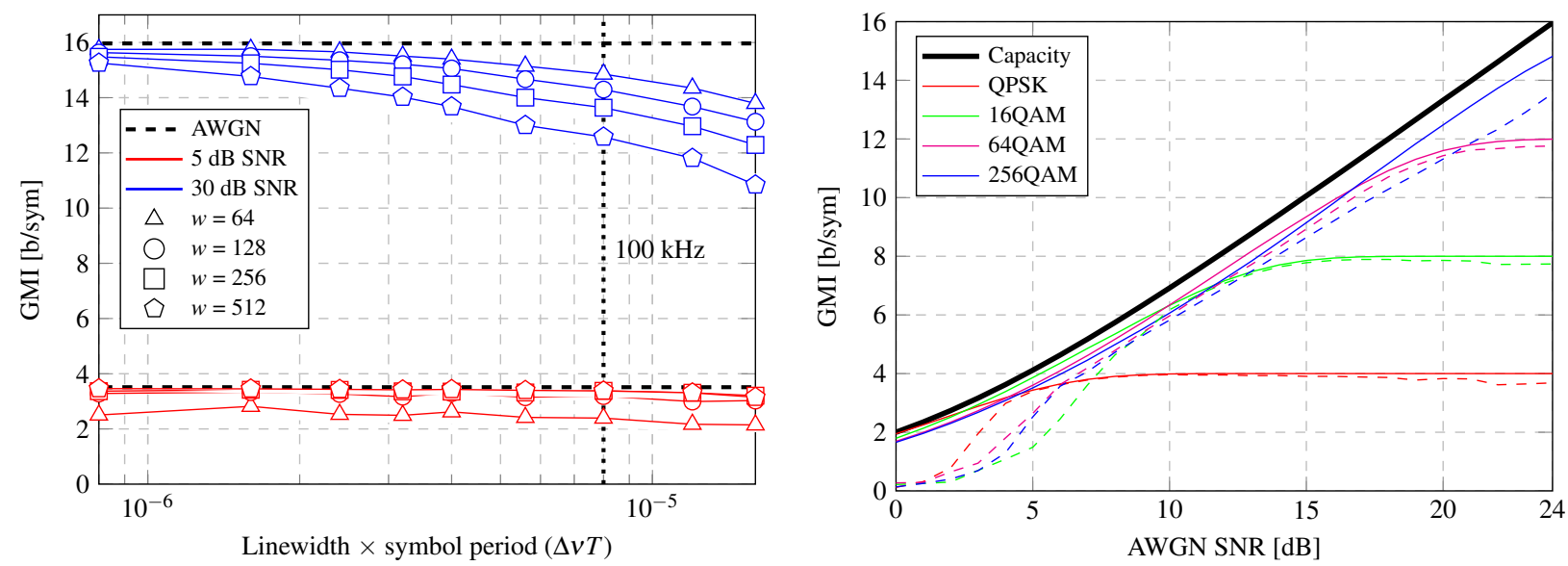

Fig. 1. a) GMI for 256QAM as a function of linewidth $\times$ symbol period product for different DD-PLL windows $w$ at SNRs of 5 and $30 \mathrm{~dB}$. b) GMI as a function of SNR without linewidth (solid lines) and with $100 \mathrm{kHz}$ linewidth (dashed lines) compensated with DD-PLL with a window $w$ of 64 symbols.

\section{Results and discussion}

First the performance of the DD-PLL algorithm was evaluated using 256QAM. Fig. 1(a) illustrates the performance for different window length $w$ at two different SNR values of 5 and $30 \mathrm{~dB}$. The results are plotted against linewidth $\times$ symbol period product $(\Delta v T)$ to make them independent of the symbol period, the corresponding linewidth values range from 10 to $200 \mathrm{kHz}$, linewidths available for commercially available laser. At an SNR of $30 \mathrm{~dB}$ the performance drops off smoothly as the linewidth increases, similar to previous reported results [1]. As expected, for larger linewidths, when phase noise is less linear, shorter windows have better performance. However, at an SNR of $5 \mathrm{~dB}$ the shorter windows have lower performance compared to longer windows where the AWGN can be averaged with larger windows. Additionally, the AIR loss with linewidth increase for the same window length varies for different SNR values. It is clear that the optimal system performance depends on multiple system parameters.

Next, the performance for multiple modulation formats was investigated with a example CPE window length. Fig. 1(b) displays the results without any linewidth and with $100 \mathrm{kHz}$ of linewidth as the AWGN SNR was varied (this SNR does not include the phase noise). To compensate the phase noise a window length $w$ of 64 symbols was chosen based on pragmatic length and tolerance to phase noise is the in high SNR region. However, even with $100 \mathrm{kHz}$ 
of linewidth there is a substantial loss in AIR, especially in very high and very low SNR regions. Although by changing the window length of the CPE algorithm the performance can be improved in certain SNR regions it would decreased in others, and similar performance trends would arise.

In Fig. 2(a) the SNR regions based on maximum AIR for an AWGN channel without phase noise are illustrated. However, once linewidth is introduced these region are shifted and changed, as shown in Fig. 2(b). It is clear that QPSK can be used for SNRs up to $6 \mathrm{~dB}$, compared to the AWGN channel were a switch would be required at $2.5 \mathrm{~dB}$. One interesting phenomena is that at this point the optimal modulation format is 64QAM instead of 16QAM (because of using the same window for all modulation formats), and the gain is substantial from 2.5 to $3.6 \mathrm{~b} / \mathrm{sym}$ ( $40 \%$ increase). Between 9.2 and 11.5 dB 16QAM does become the optimal format, however the maximum gain in that region is only from 6 to $6.2 \mathrm{~b} / \mathrm{sym}$ ( $3 \%$ increase). Thus if the code rate can be adapted the modulation order should be increase to 64QAM at much lower SNR values compared to a purely AWGN channel. Finally, beyond 20.3 dB SNR 256QAM becomes the optimal format as expected. Additionally, another difference is the range of code rates, for the AWGN channel overheads start at $\sim 10 \%$ to $195 \%$, while for phase noise channel they vary from $5 \%$ to $230 \%$. It is clear the optimal regions depends greatly on the phase noise as well as the CPE algorithms and their optimization.
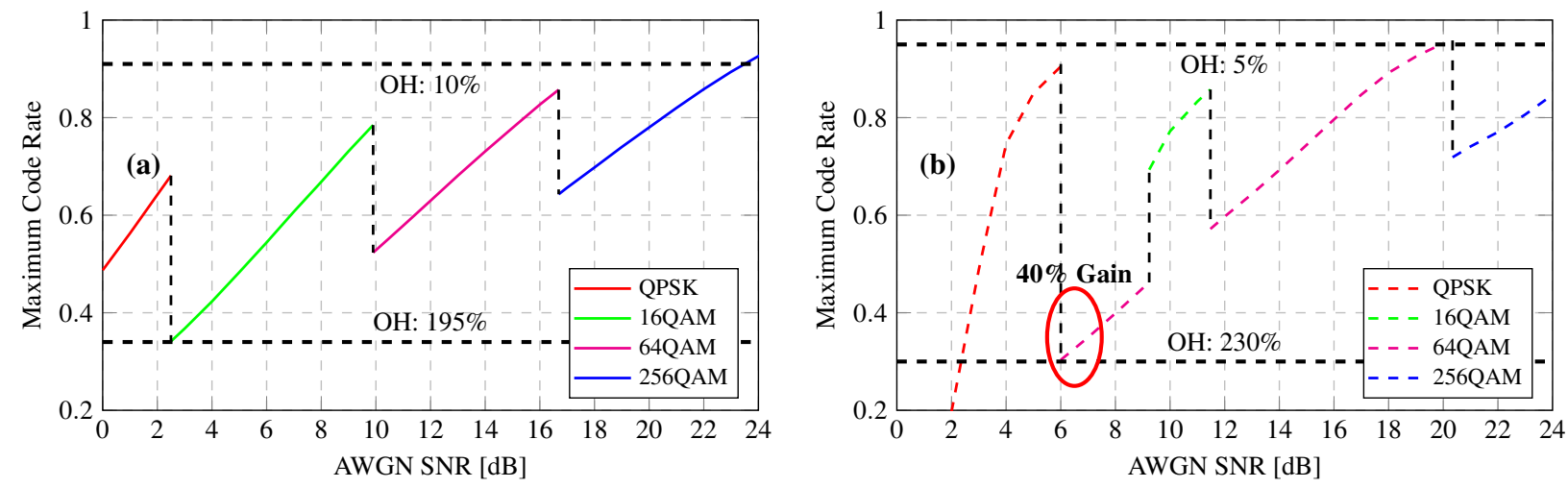

Fig. 2. Operational range for different modulation formats (a) without linewidth and (b) with $100 \mathrm{kHz}$ linewidth compensated with DD-PLL with a window $w$ of 64 symbols.

\section{Conclusions}

We have shown that the phase noise and the associated CPE outside its nominal AWGN region can significantly decrease the AIR. Although the operational regions presented in this work are only valid for this particular system under test it serves to illustrate the importance of including specific CPE and potentially other DSP algorithms in the full system design in order to maximize system throughput.

\section{References}

[1] S. M. Bilal, G. Bosco, J. Cheng, A. P. Lau, and C. Lu, "Performance and Complexity Comparison of CPE Algorithms for 256-QAM Optical Signals," in Proc. Optical Fiber Communication Conference (OFC), 2015, p. W1E.6.

[2] X. Zhou, "An Improved Feed-Forward Carrier Recovery Algorithm for Coherent Receivers With M-QAM Modulation Format," IEEE Photon. Technol. Lett., vol. 22, no. 14, pp. 1051-1053, Jul 2010.

[3] T. Pfau, S. Hoffmann, and R. Noe, "Hardware-Efficient Coherent Digital Receiver Concept With Feedforward Carrier Recovery for M-QAM Constellations," J. Lightw. Technol., vol. 27, no. 8, pp. 989 -999, Apr 2009.

[4] I. Fatadin, D. Ives, and S. Savory, "Laser Linewidth Tolerance for 16-QAM Coherent Optical Systems Using QPSK Partitioning," IEEE Photon. Technol. Lett., vol. 22, no. 9, pp. 631 -633, May 2010.

[5] R. Maher, A. Alvarado, D. Lavery, and P. Bayvel, "Increasing the information rates of optical communications via coded modulation: a study of transceiver performance," Sci. Rep., vol. 6, 2016.

[6] M. Taylor, "Phase Estimation Methods for Optical Coherent Detection Using Digital Signal Processing," J. Lightw. Technol., vol. 27, no. 7, pp. $901-914$, Apr 2009.

[7] H. Cramér, Mathematical Methods of Statistics. Princton University Press, 1999.

[8] A. Alvarado, E. Agrell, D. Lavery, R. Maher, and P. Bayvel, "Replacing the Soft-Decision FEC Limit Paradigm in the Design of Optical Communication Systems," J. Lightw. Technol., vol. 33, no. 20, pp. 4338-4352, Oct 2015.

[9] T. Koike-Akino, D. S. Millar, K. Kojima, and K. Parsons, "Phase Noise-Robust LLR Calculation with Linear/Bilinear Transform for LDPCCoded Coherent Communications," in Proc. Conference on Lasers and Electro-Optics (CLEO), May 2015, p. SW1M.3.

[10] T. Fehenberger, T. A. Eriksson, A. Alvarado, M. Karlsson, E. Agrell, and N. Hanik, "Improved Achievable Information Rates by Optimized Four-Dimensional Demappers in Optical Transmission Experiments," in Proc. Optical Fiber Communication Conference (OFC), 2016, p. W1I.4. 